\title{
Key anatomical landmarks for middle fossa surgery: a surgical anatomy study
}

\author{
Maria Peris-Celda, MD, PhD, ${ }^{1}$ Avital Perry, MD, ${ }^{1}$ Lucas P. Carlstrom, MD, PhD, ${ }^{1}$ \\ Christopher S. Graffeo, MD, ${ }^{1}$ Colin L. W. Driscoll, MD, ${ }^{1,2}$ and Michael J. Link, MD ${ }^{1,2}$ \\ Departments of ${ }^{1}$ Neurologic Surgery and ${ }^{2}$ Otolaryngology-Head and Neck Surgery, Mayo Clinic, Rochester, Minnesota
}

\begin{abstract}
OBJECTIVE Middle fossa surgery is challenging, and reliable surgical landmarks are essential to perform accurate and safe surgery. Although many descriptions of the middle fossa components have been published, a clinically practical description of this very complex anatomical region is lacking. Small structure arrangements in this area are often not well visualized or accurately demarcated with neuronavigation systems. The objective is to describe a "roadmap" of key surgical reference points and landmarks during middle fossa surgery to help the surgeon predict where critical structures will be located.
\end{abstract}

METHODS The authors studied 40 dry skulls ( 80 sides) obtained from the anatomical board at their institution. Measurements of anatomical structures in the middle fossa were made with a digital caliper and a protractor, taking as reference the middle point of the external auditory canal (MEAC). The results were statistically analyzed.

RESULTS The petrous part of the temporal bone was found at a mean of $16 \mathrm{~mm}$ anterior and $24 \mathrm{~mm}$ posterior to the MEAC. In $87 \%$ and $99 \%$ of the sides, the foramen ovale and foramen spinosum, respectively, were encountered deep to the zygomatic root. The posterior aspect of the greater superficial petrosal nerve (GSPN) groove was a mean of $6 \mathrm{~mm}$ anterior and $25 \mathrm{~mm}$ medial to the MEAC, nearly parallel to the petrous ridge. The main axis of the IAC projected to the root of the zygoma in all cases. The internal auditory canal (IAC) porus was found $5.5 \mathrm{~mm}$ lateral and $4.5 \mathrm{~mm}$ deep to the lateral aspect of the trigeminal impression along the petrous ridge (mean measurement values). A projection from this point to the middle aspect of the root of the zygoma, being posterior to the GSPN groove, could estimate the orientation of the IAC.

CONCLUSIONS In middle fossa approaches, the external acoustic canal is a reliable reference before skin incision, whereas the zygomatic root becomes important after the skin incision. Deep structures can be related to these 2 anatomical structures. An easy method to predict the location of the IAC in surgery is described. Careful study of the preoperative imaging is essential to adapt this knowledge to the individual anatomy of the patient.

https://thejns.org/doi/abs/10.3171/2018.5.JNS1841

KEYWORDS arcuate eminence; greater petrosal nerve; internal auditory canal; middle fossa surgery; surgical anatomy

$\mathrm{T}$ HE middle cranial fossa encompasses one of the most intriguing and complicated anatomical areas in skull base surgery. The middle fossa comprises the area between the sphenoid ridge anteriorly and the petrous ridge posteriorly. Its concave shape is designed to cradle the temporal lobe. Numerous neurovascular structures pass through the middle fossa, and the anatomy is further complicated by the structures contained within the temporal bone. Several anatomical studies have been performed in this region from the classical and surgical standpoint. 9,18,24,26 However, a clearly understandable study of practical, anatomical references to guide the surgeon in this region is lacking. The objective of this study is to describe a clear "roadmap" of surgical anatomical structures in the middle fossa in order to aid the skull base surgeon precisely and accurately, particularly in identifying the internal auditory canal (IAC).

\section{Methods}

Forty dry skulls ( 80 sides) from the anatomical board at our institution were used for measurements. The study was previously approved by the biospecimens subcommittee of our institutional review board. One side was excluded from

ABBREVIATIONS AE = arcuate eminence; $E A C=$ external auditory canal; $F O=$ foramen ovale; $F S=$ foramen spinosum; GSPN = greater superficial petrosal nerve; $I A C=$ internal auditory canal; MEAC = middle point of the EAC.

SUBMITTED January 6, 2018. ACCEPTED May 29, 2018.

INCLUDE WHEN CITING Published online November 9, 2018; DOI: 10.3171/2018.5.JNS1841. 
measurements from the external auditory canal (EAC) opening because of ear canal malformation. Measurements were taken with a digital caliper and a protractor. Measurements from the middle point of the EAC (MEAC) opening to osseous references in the skulls were recorded. Additionally, the lateral projections in the skull of the petrous apex, posterior aspect of the greater superficial petrosal nerve (GSPN) groove, middle point of the foramen ovale (FO), middle point of the foramen spinosum (FS), and middle point of the arcuate eminence (AE) were studied in relationship with the MEAC. The distance between the lateral aspect of the middle fossa (lateral aspect of the temporal bone squama) and these anatomical references was measured (Table 1, Fig. 1). The relationship between the EAC and the IAC, the angles between the IAC and the GPSN, and the orientation of the main axis of the IAC in relationship to the zygomatic root were studied. A straight 1-mm metallic probe was used to aid with the projections and angle measurements involving the IAC and the EAC. The IAC opening in the posterior fossa in relationship to the trigeminal impression in the petrous ridge was studied. Transillumination techniques were utilized to study the tegmen tympani area. Two formalin-fixed specimens were used to illustrate the anatomy and the technique to find the IAC in the middle fossa. Descriptive statistics of the anatomical variables (mean and range) are reported.

\section{Results}

The middle fossa, between the anterior and posterior cranial fossae, is a concave structure formed by a medial part and paired lateral parts. The medial part is centered on the sella turcica. The lateral part has an anterior wall and a floor. The anterior wall is formed by the sphenoid ridge superiorly, which belongs to the lesser sphenoid wing, and the anterior aspect of the greater sphenoid wing inferiorly. The floor is an almost flat surface tilted anteriorly and formed by part of the greater sphenoid wing, a portion of the temporal bone squama, and the superior aspect of the petrous part of the temporal bone. The posterior limit of the middle fossa is the petrous ridge, where the petrous temporal bone continues inferiorly at an approximately $90^{\circ}$ angle and forms the lateral border of the posterior fossa (Figs. 1 and 2).

The zygomatic root forms part of the temporal bone and is the posterior attachment of the zygoma. It is located immediately anterior to the EAC opening and has an irregular triangular shape seen from above, with its wide base attached to the squamous temporal bone. In all skulls studied, we found that if we drew a line between the IAC opening and the EAC opening, both openings could be found in the same coronal plane. This means that if we could look into the EAC laterally through the tissue, we would find part of the IAC opening in depth. This coronal plane usually passed through the posterior aspect of the EAC and the anterior aspect of the IAC. The main axes of the EAC and the IAC followed different angles and thus did not align perfectly (Fig. 3A).

The petrous part of the temporal bone in the middle fossa has the shape of an irregular triangle when viewed from above (Fig. 2A). The posterior limb is formed by
TABLE 1. Measurements

\begin{tabular}{lc}
\hline \multicolumn{1}{c}{ Measurement } & Mean (range) \\
\hline$A^{*}[\mathrm{~A}]$ & $36.6(27.1-43.9)$ \\
\hline $\mathrm{B} \dagger[\mathrm{B}]$ & $24(11.9-38.1)$ \\
\hline $\mathrm{C} \neq[\mathrm{C}]$ & $39.6(29-47.8)$ \\
\hline $\mathrm{S}[\mathrm{D}]$ & $23.5(18.9-27.1)$ \\
\hline PPP-MEAC & $24(16.9-32.5)$ \\
\hline APP-MEAC & $15.8(7.2-23.5)$ \\
\hline AE-MEAC & $6.6(2.4-11.6)$ \\
\hline GSPN-MEAC [1] & $6.3(1.6-14)$ \\
\hline FS-MEAC [2] & $14.7(5.8-20.9)$ \\
\hline FO-MEAC [3] & $20.6(9.6-29)$ \\
\hline TRIG-IAC [4] & $5.5(2.2-10.6)$ \\
\hline LAT-FO [5] & $30.1(24.4-39.8)$ \\
\hline LAT-FS [6] & $25.6(17.8-33.1)$ \\
\hline LAT-GSPN [7] & $25.4(14.7-33.1)$ \\
\hline LAT-AE [8] & $20.2(15-29)$ \\
\hline VERTICAL IAC [9] & $4.5(2.9-6.9)$ \\
\hline LAT-IAC & $34.5(26.6-42.2)$ \\
\hline IAC-GSPN MIN [m] & $39^{\circ}\left(20^{\circ}-60^{\circ}\right)$ \\
\hline IAC-GSPN MAX [M] & $78.6^{\circ}\left(45^{\circ}-90^{\circ}\right)$ \\
\hline AZR-FO & $2.9(-6$ to 12.4)ף \\
\hline AZR-FS & $8.8(-1.3$ to 16.2)ף \\
\hline AZR-GSPN & $17.3(4.9-24.1)$ \\
\hline AZR-APP & $7.7(-2.2$ to 15.9)ף \\
\hline
\end{tabular}

$\mathrm{AE}=$ arcuate eminence; $\mathrm{APP}=$ anterior petrosal point; $\mathrm{AZR}=$ anterior point of the zygomatic root; $F O=$ foramen ovale; $F S=$ foramen spinosum; $G S P N=$ posterior aspect of the greater petrosal nerve groove; IAC = internal auditory canal; IAC-GSPN MAX = maximum angle between the IAC and the GSPN; IAC-GSPN MIN = minimum angle between the IAC and the GSPN; LAT = lateral aspect of the craniotomy; PPP = posterior petrosal point; TRIG = lateral aspect of the trigeminal impression; VERTICAL IAC = vertical distance or depth from the petrous ridge to the IAC.

Values are in millimeters except for IAC-GSPN MIN and IAC-GSPN MAX, which are in degrees, as indicated. The numbers and letters in brackets (e.g., [A]) correspond to the references in green in Fig. 1.

* Distance from the MEAC to the anterior aspect of the squamous suture. $\dagger$ Distance from the MEAC to the projection of the posterior aspect of the squamous suture.

$\ddagger$ Distance from the superior aspect of the EAC to the superior aspect of the squamous suture.

$\S$ Distance from the MEAC to the anterior aspect of the zygomatic root. I Negative numbers mean that the structure was found anterior to the anterior point of the zygomatic root.

the petrous ridge, which has 3 important implications: it is the limit between the middle and posterior fossa, the location of the superior petrosal sinus, and the attachment of the tentorium. We can delineate an imaginary line from the posterior aspect of the zygomatic root to the petrous apex to define the anterior limb of this triangle (Fig. 2A). The lateral limb of the triangle is located between what we named the posterior petrosal point (seen from above, the most superior and posterior point of the petrous ridge; Figs. 1 and 2) and the posterior aspect of the zygomatic root. The anterior and posterior lines meet at the anterior vertex of this triangle, the petrous apex, in which the pe- 

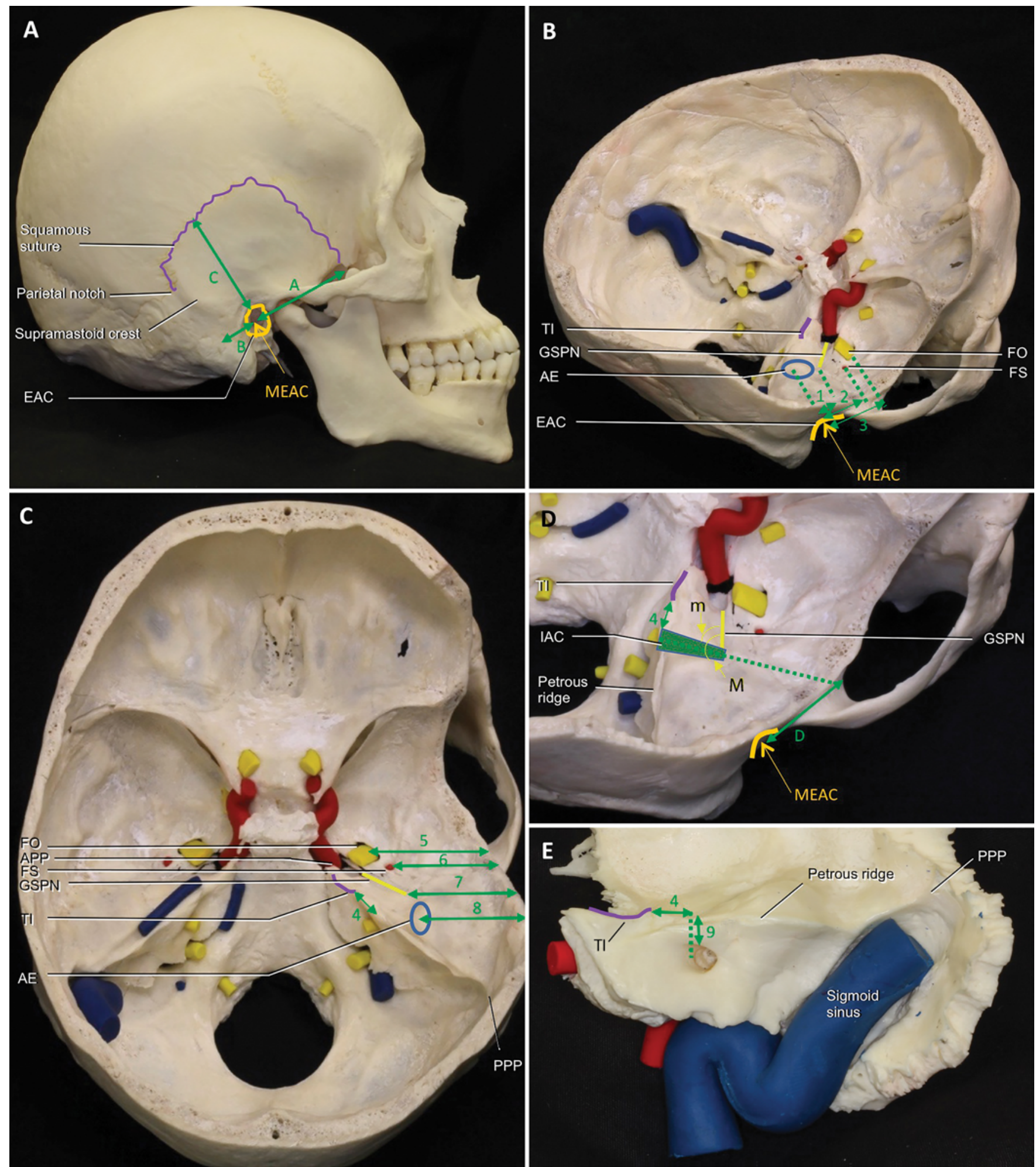

FIG. 1. Illustration of the bone anatomy and some of the measurements. The numbers and letters correspond to the measurement identifiers displayed in brackets in Table 1. A: Lateral view of the skull. The MEAC has been referenced as the middle point of the EAC. Measurements A, B, and C are represented. B: Oblique view of the skull base; the AE, GSPN, and trigeminal impression (TI) have been referenced. The green dashed lines indicate the lateral projections for measurements 1,2, and 3. C: Superior view of the skull base showing measurements in depth from the lateral edge of the craniotomy to several anatomical structures. D: IAC and relationships with the GSPN and zygomatic root. The green trapezoid area indicates the IAC with its lateral and medial borders, which have a different relationship with the GSPN, represented with the yellow line (m indicates the minimal angle with the GSPN, taking into account the medial border of the canal; M indicates the maximal angle with the GSPN, taking into account the lateral border of the canal). The green dotted line is the projection of the main axis of the IAC, which in this case projects toward the anterior aspect of the zygomatic root; in other cases it projected toward the middle or the posterior aspect of the zygomatic root. FIG. 1. (continued) $\rightarrow$ 
FIG. $1 \mathrm{E}$ : Posterior view of a right temporal bone. The measurements of the IAC performed in the posterior fossa have been illustrated. Note the posterior petrosal point (PPP) and its relationships with the sigmoid sinus. APP = anterior petrosal point. Copyright Maria Peris-Celda. Published with permission.

trous segment of the internal carotid artery ascends and enters the cavernous sinus.

\section{Anatomical Structures in the Petrous Part of the Temporal Bone}

The petrous apex was located at a mean of $16 \mathrm{~mm}$ anterior (range $7.2-23.5 \mathrm{~mm}$ ) to the MEAC and the poste- rior petrosal point at a mean of $24 \mathrm{~mm}$ posterior (range $16.09-32.5 \mathrm{~mm}$ ) to the MEAC, so the majority of the petrosal part of the temporal bone was found posterior to the MEAC. The anterior aspect of the zygomatic root was $23.5 \mathrm{~mm}$ anterior (range $18.9-27.1 \mathrm{~mm}$ ) to the MEAC and therefore anterior to the petrous apex.

The AE varied greatly in size and shape, and its middle
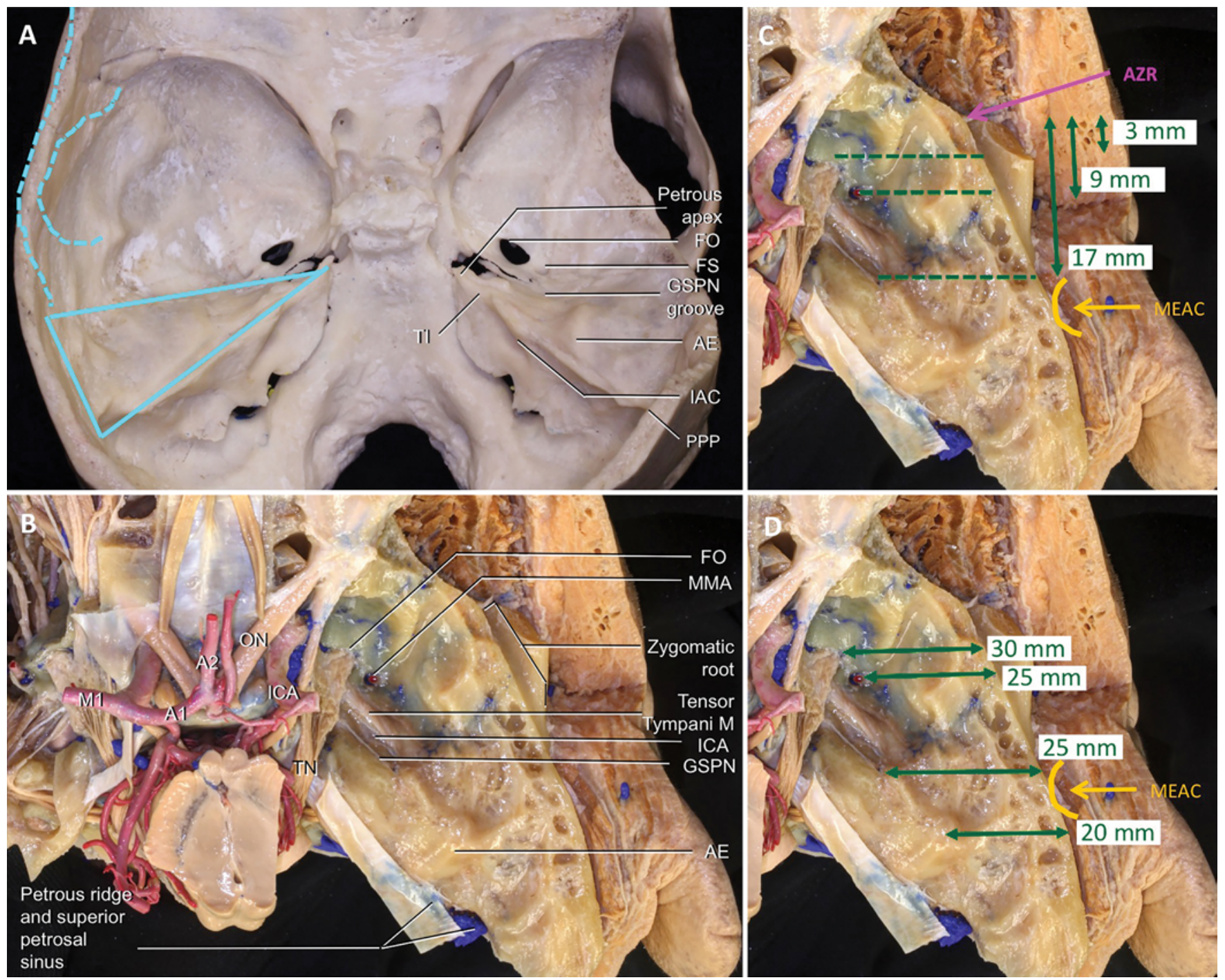

FIG. 2. Superior views of the middle fossa in a dry skull $(A)$ and formalin-fixed anatomical specimen (B-D). A: Key anatomical references for middle fossa approaches have been labeled on the right side. On the left side, the isosceles triangle that forms the superior aspect of the petrous temporal bone has been illustrated with blue lines, and a "see-through" vision of the zygoma with blue dashed lines. B: Superior view of an anatomical dissection of the middle fossa after removal of the middle fossa dura mater. C: Detail of the dissection in B, measurements of some of the key anatomical structures of the middle fossa (FO, FS, GSPN groove; see references in $B$ ) in relation to the anterior aspect of the zygomatic root (AZR). The measurements represent the mean values, not the measurements in this anatomical specimen. D: Detail of the dissection in $B$, measurements in the coronal plane. The measurements represent the mean values, not the measurements in this anatomical specimen. ICA = internal carotid artery; $\mathrm{M}=$ muscle; $\mathrm{MMA}=$ middle meningeal artery; $\mathrm{ON}=$ optic nerve; $\mathrm{TN}=$ trigeminal nerve. Copyright Maria Peris-Celda. Published with permission. 

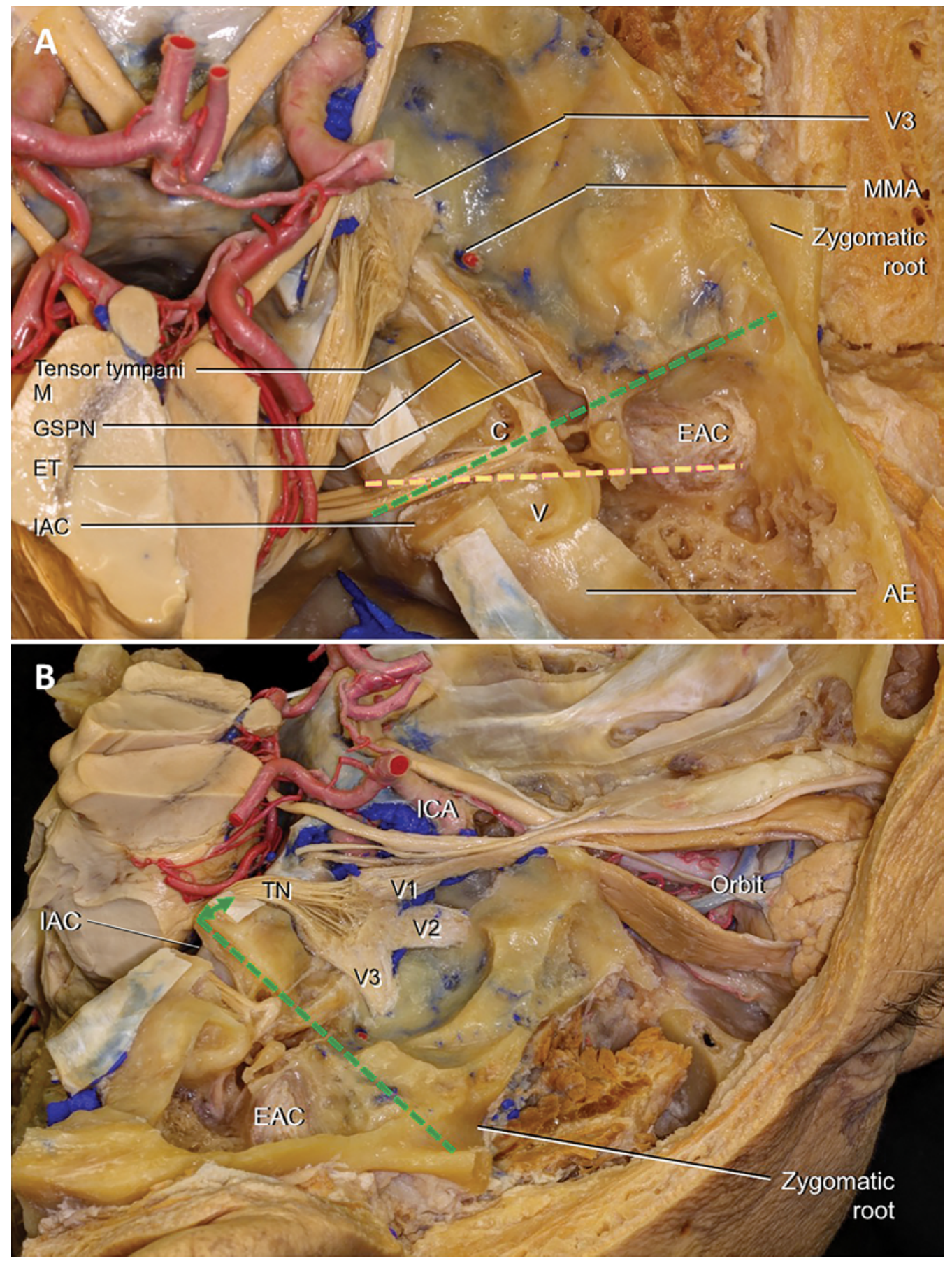

FIG. 3. Dissection of the right middle fossa. A: The IAC and the EAC have been unroofed. In all the studied specimens, there was a coronal plane that passed through the posterior aspect of the EAC and the anterior aspect of the IAC (orange dashed line). In this specimen, the main axis of the IAC projects toward the posterior third of the zygomatic root (green dashed line). B: The proposed method to estimate the medial aspect of the IAC is finding a point $5 \mathrm{~mm}$ lateral to the lateral aspect of the trigeminal nerve and trigeminal impression in the petrous ridge and projecting a line from this point toward the middle aspect of the zygomatic root. If this line crosses the GSPN, it must be directed toward the posterior aspect of the zygomatic root. The drilling would start at this point or slightly medial, at the petrous apex, until the IAC dura mater is found. $\mathrm{C}=$ cochlea; $E T=$ eustachian tube; $V=$ vestibular labyrinth, semicircular canals. Copyright Maria Peris-Celda. Published with permission.

aspect was a mean of $6.5 \mathrm{~mm}$ posterior (range 2.4-11.6 $\mathrm{mm}$ ) to the MEAC and $20 \mathrm{~mm}$ medial (range 15-29 $\mathrm{mm}$ ) to the lateral aspect of the temporal bone.

The groove/hiatus for the GSPN in the petrous part of the temporal bone marked the superficial portion of this nerve in the middle fossa. The groove was almost parallel to the petrous ridge, and the most posterior part of the groove could be found approximately $6 \mathrm{~mm}$ anterior 
(range $1.6-14 \mathrm{~mm}$ ) to the $\mathrm{MEAC}$ and $17 \mathrm{~mm}$ posterior (range 4.9-24.1 $\mathrm{mm}$ ) to the anterior part of the zygomatic root. The posterior part of the GSPN groove was $25 \mathrm{~mm}$ medial (range 14.7-33.1 mm) to the edge of the bone (external surface of the squamous temporal bone).

The thinnest translucent part of the temporal bone, which includes the tegmen tympani, was superior to the EAC in $67 \%$ of the cases and superior and posterior in $33 \%$.

\section{Temporal Bone Squama}

Looking at the skull laterally, the squamous portion of the temporal bone ended $36 \mathrm{~mm}$ anterior to the MEAC (measurement A, Fig. 1) and the posterior portion was 24 $\mathrm{mm}$ posterior (measurement B, Fig. 1). The parietal notch, the indentation of the temporal bone between the squamous part of the temporal bone and the mastoid, corresponded in $66 \%$ of the cases to the posterior petrosal point. The parietal notch in $34 \%$ of the cases corresponded to the sigmoid sinus or the transverse-sigmoid junction.

The supramastoid crest, or posteroinferior aspect of the temporal line, is an elevation of the temporal bone superior to the mastoid process, which corresponds to the posterior attachment of the temporalis muscle. The upper limit of the horizontal part of the supramastoid crest was supratentorial in $93 \%$ of the cases and infratentorial in $7 \%$. A point $1 \mathrm{~cm}$ above the horizontal aspect of the supramastoid crest was found to be supratentorial in all cases.

\section{Sphenoid Bone Structures in the Middle Fossa Floor}

The part of the greater wing of the sphenoid bone corresponding to the middle fossa floor contains the FO and the FS. The FO was a mean of $3 \mathrm{~mm}$ posterior (range -6 to $12.4 \mathrm{~mm}$; negative number means anterior to the zygomatic root) to the anterior aspect of the zygomatic root and $30 \mathrm{~mm}$ medial (range $24.4-39.8 \mathrm{~mm}$ ) to the lateral aspect of the skull. The FS was a mean of $9 \mathrm{~mm}$ posterior (range -1.3 to $16.2 \mathrm{~mm}$ ) to the anterior aspect of the zygomatic root and $25.5 \mathrm{~mm}$ medial (range 17.8-33.1 mm) (Fig. 2, Table 1$)$. In 10 cases $(12.5 \%)$, the middle point of the FO was located between 1 and $6 \mathrm{~mm}$ anterior to the anterior aspect of the zygomatic root, and only in 1 case (1.3\%) was the FS anterior to this point.

\section{Internal Auditory Canal}

The IAC is a funnel-shaped structure with a wider base at its opening in the posterior fossa (porus), becoming narrower at the fundus. The medial aspect of the porus, in the posterior fossa, was $5.5 \mathrm{~mm}$ posterolateral (range 2.2-10.6 $\mathrm{mm}$ ) to the lateral aspect of the trigeminal impression along the petrous ridge. The IAC opening was a mean of 4.5 $\mathrm{mm}$ inferior (range 2.9-6.9 $\mathrm{mm}$ ) to the petrous ridge (Fig. $1 \mathrm{E})$. Both the anteromedial and posterolateral walls of the IAC form an angle with the GSPN. The anteromedial wall formed a smaller angle (mean $39^{\circ}$, range $20^{\circ}-60^{\circ}$ ) than the posterolateral wall (mean $78.6^{\circ}$, range $45^{\circ}-90^{\circ}$ ) (Fig. 1D).

The median or main axis of the IAC projected to the anterior root of the zygoma in $39 \%$ of the cases, to the middle aspect in $31 \%$, and to the posterior aspect in $30 \%$.

\section{Discussion}

Meticulous descriptions of the middle fossa foramina and its components have been previously reported, 3,15,22,26 but few with relevant surgical anatomy, ${ }^{16,18,24}$ and to our knowledge none of them focusing on relationships between internal and external landmarks.

Middle fossa approaches are utilized to access a variety of pathologies arising in the temporal bone, middle fossa, or posterior fossa. In the latter case, it can be the access route to lesions in the IAC, such as vestibular schwannomas, or to other posterior fossa pathologies through the petrous apex. The classical middle fossa approach without frontotemporal extension exposes the bony surface of the posterior half of the middle fossa and the dura mater covering the inferior temporal lobe. Extradural retraction of the temporal lobe is necessary in order to expose the middle fossa structures and perform the operation. The posterior petrosal point is an important anatomical reference and is one of the most complex points with respect to venous anatomy in the head, as it lies just above the junction of the transverse and sigmoid sinuses. The temporal veins, including the vein of Labbé, superior petrosal sinus, and a variable arrangement of tentorial veins, drain ultimately into the transverse-sigmoid junction, in close proximity to this point ${ }^{13,19}$ Sustained retraction, which can also result in occlusion or diminished flow through these veins, can provoke temporal lobe edema or even potentially venous infarction and hemorrhage with significant, permanent neurological deficit or seizures..$^{20,23}$ Any attempt to improve the efficiency of the operation with clear anatomical landmarks may ultimately result in less operative time and retraction.

Two main external landmarks have been considered in this study, the EAC and the zygomatic root. The EAC is a good reference mainly before skin incision, whereas the zygomatic root becomes more evident and important after skin incision. Professor Rhoton pioneered the concept of "see-through vision" in neurosurgery, which refers to the ability to locate deep critical structures looking at the superficial planes with the knowledge of surgical anatomy. ${ }^{17}$ This helps the surgeon from the early stages of the operation with surgical planning, execution, and integration with surgical navigation when utilized. Surgical anatomy knowledge has to be accompanied by careful study of the pathology and anatomy of the case (see Fig. 5). Related to the results conveyed by this study, pathologies located on the superior semicircular canal, such as superior semicircular canal dehiscence, will be centered within $1.5 \mathrm{~cm}$ posterior to the MEAC. When approaching the FO or FS, in $86 \%$ of cases they will be found within the zygomatic root width in depth (at 30 and at $25 \mathrm{~mm}$ mean depth from the craniotomy edge, respectively). We found that in specimens with narrow zygomatic roots, the FO was up to 6 $\mathrm{mm}$ anterior, so it is advisable to extend the craniotomy a few millimeters anterior to the anterior aspect of the zygomatic root if exposure of the FO is required. The lateral projection of the petrous part of the temporal bone (from the apex to the posterior petrosal point) is located between the anterior aspect of the zygomatic root and a mean of 24 $\mathrm{mm}$ posterior to the MEAC. The posterior petrosal point was located in the majority of cases at the level of the parietal notch of the temporal bone. Only one of the petrous apices was slightly $(2.2 \mathrm{~mm})$ anterior to the anterior aspect of the zygomatic root (Figs. 2 and 3). 
The GSPN groove is contained in the petrous portion of the temporal bone. The posterior aspect of the GSPN groove can be found in the middle fossa after dural elevation in most cases at a variable distance from the geniculate ganglion going toward the petrous apex..$^{14,26}$ This variability can range from being completely uncovered, with the geniculate ganglion exposed, to being completely covered in bone. ${ }^{6}$ In all the skulls analyzed, the GSPN groove could be identified (Fig. 2). The horizontal segment of the carotid artery is inferior and parallel to the GSPN inside the petrous part of the temporal bone, and the trajectory of the GSPN is almost parallel to the petrous ridge. The geniculate ganglion can be localized at the point where the line of the GSPN groove and the IAC meet. This is the point where the GSPN leaves the geniculate ganglion. The labyrinth is posterolateral to the geniculate ganglion, and the cochlea is inferomedial. There have been numerous descriptions of how to find the GSPN and how to dissect it, anterior to posterior or posterior to anterior. ${ }^{8}$ Most authors start the dissection posterior to anterior. In this way, the posterior aspect is closer to the edge of the craniotomy, and it is easier to separate the nerve from the overlying dura working in this direction. The posterior aspect of the GSPN was found between the MEAC and the anterior aspect of the zygomatic root, specifically within the $15 \mathrm{~mm}$ anterior to the MEAC in all cases, and within the $25 \mathrm{~mm}$ medial to the craniotomy edge, but as close as $14 \mathrm{~mm}$ depending on the bony coverage and dimensions of the head.

The IAC is a funnel-shaped structure that narrows distally at the fundus. The porus is deeper (more inferior) than the fundus. A good estimation of the IAC is key in middle fossa approaches to vestibular schwannomas and in anterior petrosectomies to approach the posterior fossa via the middle fossa. There have been numerous descriptions of how to find the IAC. $1,2,5,7,10,12,21,25$ The anatomy is variable, and the exact location of the IAC may be impossible to predict in all cases with anatomical references. To our knowledge this is the first study that takes into account an extracranial structure as a landmark to find the IAC. One of the most used references to find the IAC is to bisect the main axis of the AE and the main axis of the GSPN. ${ }^{4}$ The AE is an anatomical structure that has been advocated as a reference for the superior semicircular canal, but the relationship does not apply in all cases. ${ }^{17}$ We found the AE to be highly variable and its main axis difficult to describe. The view the middle fossa approach gives of the structures is very different from the superior view of the middle fossa in anatomical dissections with the brain removed. The middle point of the AE was found $20 \mathrm{~mm}$ deep (range $15-29 \mathrm{~mm}$ ) to the edge of the craniotomy, approximately at the posterior aspect of the EAC. Due to this anatomical variation, some surgeons drill the AE until they find the blue line of the superior semicircular canal. What we propose is to dissect the middle fossa dura mater until the petrous ridge is found, which is also the first step of the technique proposed by Tanriover et al. ${ }^{24}$ The trigeminal nerve lies over the trigeminal impression and formed a very reliable landmark at the petrous ridge in all of the skulls that we examined. The senior authors (M.J.L., C.L.W.D.) have found that a safe strategy to find the IAC is to start drill- ing in the petrous apex just posterolateral to the trigeminal impression from medial to lateral until the dura mater of the posterior fossa and medial aspect of the IAC are found. In our study, the mean distance from the posterolateral aspect of the trigeminal impression to the anteromedial aspect of the IAC along the petrous ridge was $5.5 \mathrm{~mm}$, the shortest distance was $2 \mathrm{~mm}$, and the largest was $10 \mathrm{~mm}$. A line connecting this point with the middle third of the zygomatic root could estimate the orientation of the IAC (Figs. 3 and 4). This imaginary trajectory must not cross the GSPN at any point, as fibers from the geniculate ganglion form part of nervus intermedius and travel with the facial nerve. If a planned trajectory does cross the GSPN, it must be modified posteriorly such that it is directed more toward the posterior zygomatic root. The surgeon must be aware that the orientation of the IAC axis is variable and can point toward the anterior, middle, or posterior aspect of the zygomatic root (pointing toward each of these locations in approximately one-third of cases). The IAC projection to the anterior, middle, or posterior third of the zygomatic root can be easily assessed preoperatively in CT or MR images (Fig. 5). Once the anteromedial aspect of the IAC dura mater is found, it can be safely skeletonized with the diamond drill and followed toward the fundus. In order to be safe, we recommend starting the drilling $2 \mathrm{~mm}$ lateral to the lateral aspect of the trigeminal impression and proceeding along the petrous ridge until the dura mater of the IAC is found. This drilling is performed within the petrous apex, so only bone, bone marrow, or petrous apex air cells will be found. The cochlea is at the anterolateral vertex of the petrous apex, very close to the geniculate ganglion and thus away from the petrous ridge. It is important to take into consideration that the opening of the IAC can be as much as $7 \mathrm{~mm}$ deep to the petrous ridge (mean $4.5 \mathrm{~mm}$ ). These measurements can be easily assessed with a preoperative MR or CT scan.

We found that the parietal notch (indentation between the squamous suture and the mastoid) was coincident in two-thirds of the cases with the posterior petrosal point. The mean distance from the MEAC to the posterior aspect of the squamous suture and the mean distance from the MEAC to the posterior petrosal point were found to be the same. A point $1 \mathrm{~cm}$ above the horizontal aspect of the supramastoid crest was found to be supratentorial in all cases. In order to place the posterior burr hole in a middle fossa craniotomy, we recommend the location immediately anterior to the posterior aspect of the squamous suture and $1 \mathrm{~cm}$ superior to the supramastoid crest. The dura mater can be dissected inferiorly to feel the floor of the middle fossa and adjust the inferior aspect of the craniotomy as needed. This will avoid placing burr holes in undesired locations, such as the transverse-sigmoid junction, above the tentorium posterior to the petrous bone, or over the mastoid.

A limitation of our anatomical study of dry skulls is that we did not know the sex or ethnicity of the specimens, and this may potentially alter the applicability of our findings. Although the measurements can be variable, the location in relation to external structures is nearly constant. We strongly advocate that careful study of preoperative examinations is essential in any neurosur- 

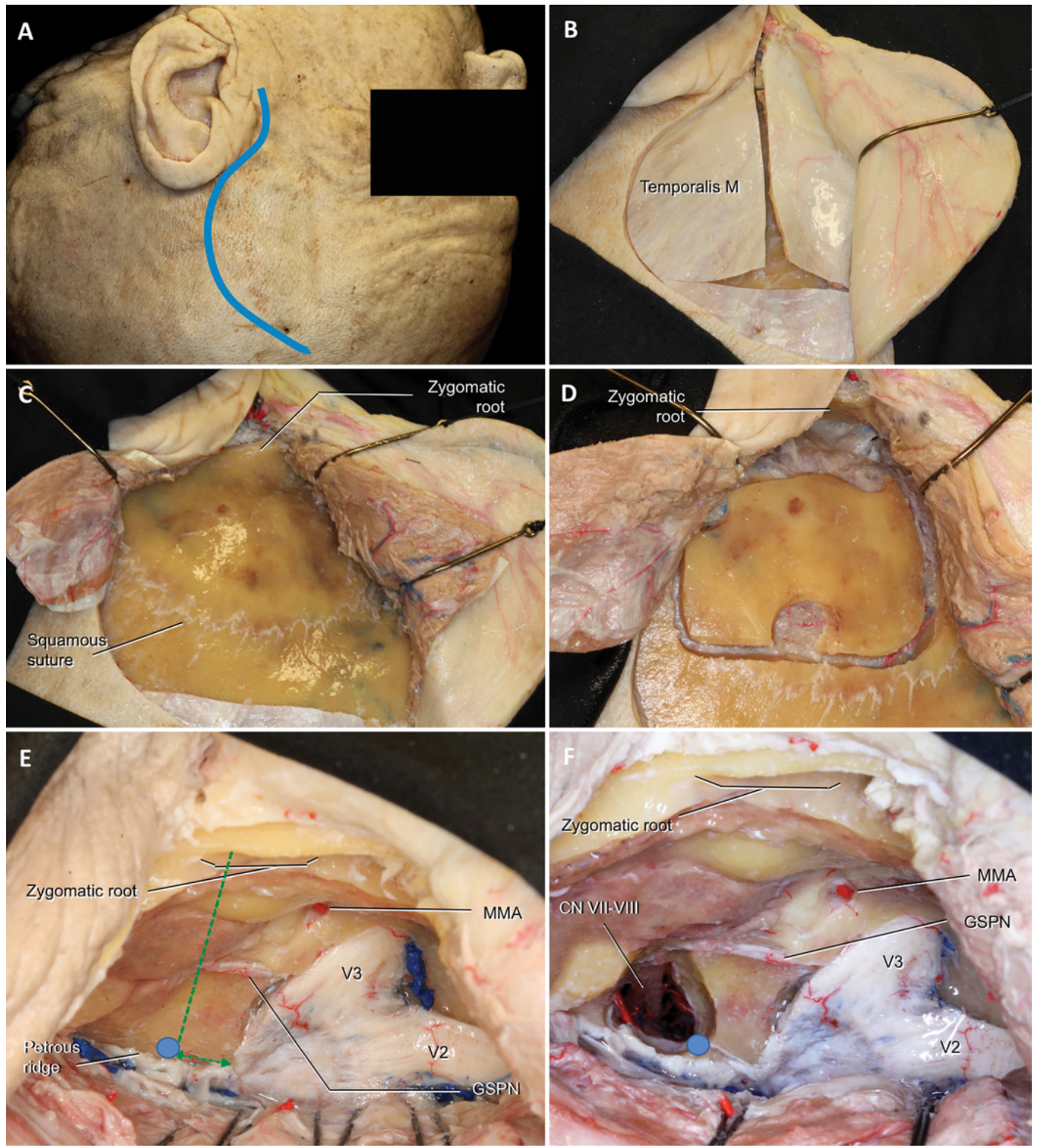

FIG. 4. Left middle fossa approach and opening of the IAC in a formalin-fixed specimen. A: Skin incision. B: The cutaneous flap was reflected anteriorly and the temporalis muscle divided in T. C: Exposure of the zygomatic root and the squamous part of the temporal bone. D: Craniotomy. E: Exposure of the middle fossa floor with retraction of the temporal lobe and dura mater to find the petrous ridge. The projection of the medial aspect of the IAC was estimated with a line passing through a point $5 \mathrm{~mm}$ lateral to the trigeminal nerve (trigeminal impression) at the petrous ridge (blue dot) toward the middle third of the zygomatic root, slightly modified posteriorly to avoid crossing the GSPN (green dotted line). F: Exposure of cranial nerves (CNs) VII-VIII in the IAC through the middle fossa. Drilling was started at the estimated point in the petrous apex until the medial aspect of the IAC dura mater was found. In this specimen, the main axis of the canal projected toward the posterior aspect of the zygomatic root. The estimated starting point and projection were useful to find the medial aspect of the IAC dura mater. $M=$ muscle; $M M A=$ middle meningeal artery. Copyright Maria Peris-Celda. Published with permission. 

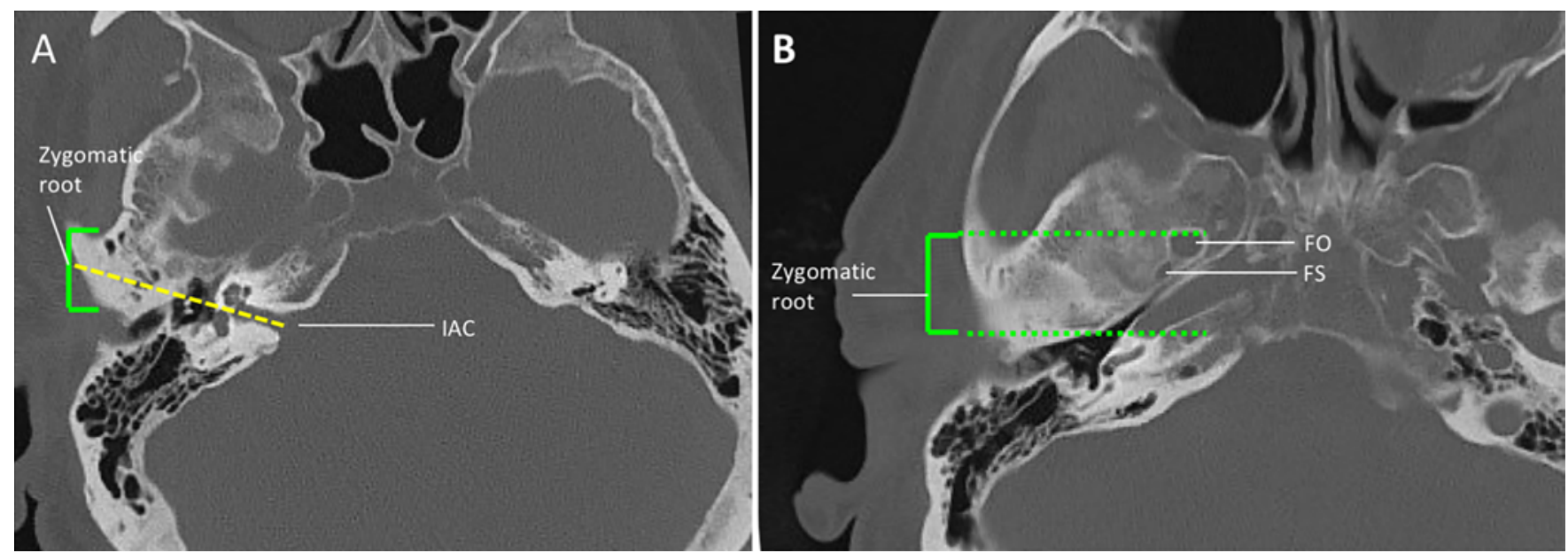

FIG. 5. Example of some of the anatomical relationships that can be studied in preoperative examinations to adapt surgical anatomy knowledge to the patient's individual anatomy. A: Axial CT scan. In this case, the main axis of the IAC projects toward the middle third of the zygomatic root. B: Axial CT scan. The FS and FO are located in depth within the width of the zygomatic root. In our study this relationship was present in $86.4 \%$ of cases for FO and in $98.8 \%$ for the FS.

gical operation in order to adapt the previously acquired general anatomical knowledge to the particular anatomy of the patient and the pathology. We also consider neuronavigation a valuable component, although it may not always be accurate throughout the operation, and thus its use should not be a substitute for but rather a complement to anatomical knowledge. ${ }^{11}$ Furthermore, neuronavigation has not been able, as of yet, to locate small structures such as the GSPN groove, and the typical margin of error with registration makes it not particularly useful in identifying such close anatomical arrangements during middle fossa surgery. Our intention for this study was to help the skull base surgeon to reliably expect where the structures will be located, potentially increasing the efficacy of the procedure; we also believe that the anatomical knowledge acquired in this study can be a valuable tool when utilized in conjunction with neuronavigation. Although Professor Rhoton embraced and studied neuronavigation techniques,${ }^{11}$ he also considered that "the best navigation is the surgical anatomy in the mind of the neurosurgeon in order to make surgery more accurate, gentle, and safe for our patients" (A. Rhoton, personal communication, 2011).

\section{Conclusions}

In middle fossa surgery, the EAC is a good anatomical reference, especially before skin incision, whereas the zygomatic root becomes important after the skin incision. In most cases, both the FO and the FS will be encountered deep to the zygomatic root. The main axis of the IAC projects toward the zygomatic root. A line connecting a point $5 \mathrm{~mm}$ lateral to the trigeminal impression along the petrous ridge and the middle point of the zygomatic root could predict the location and orientation of the IAC. Pathologies often displace or distort normal anatomy, and detailed knowledge of these relationships in conjunction with thorough study of preoperative imaging studies is essential in planning and tackling these lesions.

\section{Acknowledgments}

We would like to acknowledge the late Dr. Albert L. Rhoton, director of "The Microscopic and Endoscopic Neurosurgical Laboratory" at the University of Florida, Gainesville, where the photographs in Figs. 1-3 were taken by the first author (M.P.C.).

\section{References}

1. Catalano PJ, Eden AR: An external reference to identify the internal auditory canal in middle fossa surgery. Otolaryngol Head Neck Surg 108:111-116, 1993

2. Cokkeser Y, Aristegui M, Naguib MB, Saleh E, Taibah AK, Sanna M: Identification of internal acoustic canal in the middle cranial fossa approach: a safe technique. Otolaryngol Head Neck Surg 124:94-98, 2001

3. Edwards B, Wang JM, Iwanaga J, Loukas M, Tubbs RS: Cranial nerve foramina part I: a review of the anatomy and pathology of cranial nerve foramina of the anterior and middle fossa. Cureus 10:e2172, 2018

4. Garcia-Ibanez E, Garcia-Ibanez JL: Middle fossa vestibular neurectomy: a report of 373 cases. Otolaryngol Head Neck Surg 88:486-490, 1980

5. House WF: Surgical exposure of the internal auditory canal and its contents through the middle, cranial fossa. Laryngoscope 71:1363-1385, 1961

6. Isaacson B, Vrabec JT: The radiographic prevalence of geniculate ganglion dehiscence in normal and congenitally thin temporal bones. Otol Neurotol 28:107-110, 2007

7. Jackler RK, Gladstone HB: Locating the internal auditory canal during the middle fossa approach: an alternative technique. Skull Base Surg 5:63-67, 1995

8. Jittapiromsak P, Sabuncuoglu H, Deshmukh P, Nakaji P, Spetzler RF, Preul MC: Greater superficial petrosal nerve dissection: back to front or front to back? Neurosurgery 64 (5 Suppl 2):253-259, 2009

9. Kakizawa Y, Hongo K, Rhoton AL Jr: Construction of a three-dimensional interactive model of the skull base and cranial nerves. Neurosurgery 60:901-910, 2007

10. Kartush JM, Kemink JL, Graham MD: The arcuate eminence. Topographic orientation in middle cranial fossa surgery. Ann Otol Rhinol Laryngol 94:25-28, 1985

11. Komune N, Matsushima K, Matsuo S, Safavi-Abbasi S, Matsumoto N, Rhoton AL Jr: The accuracy of an electromagnetic 
navigation system in lateral skull base approaches. Laryngoscope 127:450-459, 2017

12. Lee HK, Kim IS, Lee WS: New method of identifying the internal auditory canal as seen from the middle cranial fossa approach. Ann Otol Rhinol Laryngol 115:457-460, 2006

13. Matsushima T, Rhoton AL Jr, de Oliveira E, Peace D: Microsurgical anatomy of the veins of the posterior fossa. J Neurosurg 59:63-105, 1983

14. Osawa S, Rhoton AL Jr, Seker A, Shimizu S, Fujii K, Kassam AB: Microsurgical and endoscopic anatomy of the vidian canal. Neurosurgery 64 (5 Suppl 2):385-412, 2009

15. Patil J, Kumar N, Rao MKG, Swamy RS, Somayaji SN, Nayak SB, et al: The foramen ovale morphometry of sphenoid bone in South Indian population. J Clin Diagn Res 7:2668-2670, 2013

16. Peris-Celda M, Graziano F, Russo V, Mericle RA, Ulm AJ: Foramen ovale puncture, lesioning accuracy, and avoiding complications: microsurgical anatomy study with clinical implications. J Neurosurg 119:1176-1193, 2013

17. Rhoton AL: Navigating the temporal bone [video]. Rhoton Collection (https://www.youtube.com/ watch?v=AEIKqKh752g) [Accessed July 19, 2018]

18. Rhoton AL Jr: The anterior and middle cranial base. Neurosurgery 51 (4 Suppl):S273-S302, 2002

19. Rhoton AL Jr: The cerebral veins. Neurosurgery 51 (4 Suppl):S159-S205, 2002

20. Sameshima T, Fukushima T, McElveen JT Jr, Friedman AH: Critical assessment of operative approaches for hearing preservation in small acoustic neuroma surgery: retrosigmoid vs middle fossa approach. Neurosurgery 67:640-645, 2010

21. Sampath R, Glenn C, Patil S, Vannemreddy P, Gardner L, Nanda A, et al: A novel method of identifying the internal acoustic canal in the middle fossa approach in a cadaveric study-the rule of 2s. J Neurol Surg B Skull Base 73:253260,2012

22. Sepahdari AR, Mong S: Skull base CT: normative values for size and symmetry of the facial nerve canal, foramen ovale, pterygoid canal, and foramen rotundum. Surg Radiol Anat 35:19-24, 2013

23. Stripf T, Bruehl K, Mann WJ, Amedee RG: Clinical and ra- diologic sequelae of the middle fossa approach to the internal auditory canal. Otol Neurotol 25:787-790, 2004

24. Tanriover N, Sanus GZ, Ulu MO, Tanriverdi T, Akar Z, Rubino PA, et al: Middle fossa approach: microsurgical anatomy and surgical technique from the neurosurgical perspective. Surg Neurol 71:586-596, 2009

25. Tsunoda A, Komatsuzaki A, Kobayashi M, Suzuki Y: Threedimensional image for the middle fossa approach and its anatomical considerations. Laryngoscope 111:1048-1052, 2001

26. Tubbs RS, Custis JW, Salter EG, Sheetz J, Zehren SJ, Oakes WJ: Landmarks for the greater petrosal nerve. Clin Anat 18:210-214, 2005

\section{Disclosures}

The authors report no conflict of interest concerning the materials or methods used in this study or the findings specified in this paper.

\section{Author Contributions}

Conception and design: Peris-Celda. Acquisition of data: PerisCelda, Perry, Carlstrom, Graffeo. Analysis and interpretation of data: Peris-Celda. Drafting the article: Peris-Celda. Critically revising the article: Link, Perry, Graffeo, Driscoll. Reviewed submitted version of manuscript: Link, Peris-Celda, Perry, Graffeo, Driscoll. Approved the final version of the manuscript on behalf of all authors: Link. Statistical analysis: Peris-Celda.

\section{Supplemental Information \\ Previous Presentations}

Portions of this work were presented at the 28th North American Skull Base Society Annual Meeting, Coronado, CA, February 16-18, 2018.

\section{Correspondence}

Michael J. Link: Mayo Clinic, Rochester, MN. link.michael@ mayo.edu. 